\title{
Agrobacterium rhizogenes-transformed roots of Astragalus membranaceus (Fisch. Ex Link) Bunge as a source of valuable secondary metabolites
}

\author{
Tatyana Novikova ${ }^{1 *}$, Elena Ambros, Tatyana Zheleznichenko, Yulianna Zaytseva, and \\ Yungeree Oyunbileg ${ }^{2}$ \\ ${ }^{1}$ Central Siberian Botanical Garden, Siberian Branch of RAS, 630090, Novosibirsk, Russia Russian \\ ${ }^{2}$ Institute of General and Experimental Biotechnology, Mongolian Academy of Sciences, 13330, \\ Ulaanbaatar, Mongolia
}

\begin{abstract}
In this study the results of agrobacterial transformation of Astragalus membranaceus (Fisch. Ex Link) Bunge, a medicinal plant from Mongolia and China with a wide spectrum of pharmacological action were presented. As explants for transformation, in vitro derived microshoots as well as hypocotyls, cotyledons and primary shoots isolated from seedlings were used. The most effective from two tested Agrobacterium rhizogenes strains (A4-RT, 15834 SWISS) was 15834 SWISS, causing the transformation of various types of explants, including primary shoots as a more responsive type. The optimal co-cultivation time of explants with bacterial suspension (48 hours) and the highest transformation rate of primary shoots $(36.4 \%)$ were established. PCR analysis confirmed the transgenic nature of the roots transformed with strain 15834 SWISS and the absence of bacterial contamination.
\end{abstract}

\section{Introduction}

In the past decades the growing demand for natural, renewable sources of medicinal substances has significantly increased interest in tissue culture techniques as an attractive alternative that allows enhancing the yield of target metabolites or inducing biosynthesis of new compounds without damaging the natural plant populations. The advantages of the biotechnological approach are obvious: high productivity of important plant pharmaceuticals, ease of extraction of target metabolites, guaranteed yield of target products regardless of seasonal and weather conditions, the absence of toxic substances (pesticides, herbicides and other pollutants) in crops, the ability to scale the process by the use of bioreactors [1,2]. However, despite these advantages nowadays the commercial list of producer cultures is limited to dozens [3]. The processes of biosynthesis of secondary metabolites in callus or suspension cultures occur in non-specialized proliferating cells, whereas in a plant the metabolites are produced only in organized structures [4]. Hairy root (HR) cultures induced by transformation using Agrobacterium rhizogenes have

\footnotetext{
*Corresponding author: tin27@mail.ru
} 
revolutionized the role of plant tissue culture in secondary metabolite production due to a high degree of cell differentiation, rapid growth, genetic and biochemical stability, and the ability to synthesize chemical compounds of both roots and aboveground mass $[5,6]$. The ability to synthesize their own phytohormones provides fast growth of HR in nutrient media without growth regulators, the presence of which is undesirable in the final products [5]. These advantages allow using HR cultures as an effective biotechnological platform for obtaining valuable metabolites. In recent years, the so-called civilization diseases have acquired a significant share: viral, oncological, cardiovascular, neuropsychic, allergic, etc. Therefore, research efforts are focused on obtaining drugs with antitumor and antiviral activities that increase the duration and quality of life (adaptogens, biostimulants, including immunostimulants) as well as cardiological and antimicrobial medicines [5, 7]. According to these demands, we chose Astragalus membranaceus (Fisch. Ex Link) Bunge (Leguminosae) included in the "elite" of medicinal plants of Mongolia, China and also widely used in medicine in Western Siberia, Transbaikalia, and the Far East [8 ]. It was found that the dry roots of this species (Radix astragali) possess hepatoprotective, antiinflammatory, antiviral, immunomodulatory and antihyperglycemic effects $[9,10,11]$. The main pharmacologically active components of A. membranaceus are astragalosides (AG), a variety of triterpene saponins of the cycloartan type [12]. Among them, astragaloside I (AG I), astragaloside II (AG II), iso-astragaloside II (IAH II), astragaloside III (AG III), astragaloside IV (AG IV) and cycloastragenol are the six main derivatives of astralosides (AG). Pharmacological studies have shown that AG IV, a key astralagoside, is of great interest because of its many functions, such as cardioprotective, antitumor, antiviral, immunoregulatory, anti-inflammatory, antihypertensive, hepatoprotective, antidiabetic, neuroprotective, etc. [13]. In addition to astralogosides, A. membranaceus contains typical for legume plants phytoalexins - substances of an isoflavone nature, including formononetin and calicosin, which have antioxidant, antiviral, anti-inflammatory, estrogen, neuroprotective, and hematopoietic effects [14]. The proven therapeutic activities of the secondary metabolites of $A$. membranaceus stimulate the creation of biotechnological platforms for their production in tissue culture systems. However, despite the progress made, the developed protocols for the cultivation of $A$. membranaceous HR have not reached the high level of the target metabolites production (astralogosides, isoflavonoids) sufficient for their commercial application [12].

The objectives of this study were (i) to optimize the transformation of A. membranaceus using two strains of Agrobacterium rhizogenes; (ii) to obtain various HR lines; and (iii) to confirm the transgenic nature of HR cultures using PCR analysis

\section{Materials and Methods}

\subsection{Explant source and culture conditions}

For genetic transformation in vitro derived microshoots of $A$. membranaceus supported on the Murasige-Skoog medium [15] and seedlings obtained from mature seeds collected by O.V. Kotsupiy in 2000 in the vicinity of Adon-Chelon (Trans-Baikal Territory) were used (Fig. 2). To overcome exogenous physical dormancy, mechanical scarification was carried out using sandpaper, then the scarified seeds were sterilized with $70 \%$ ethanol solution (2 $\mathrm{min}$ ), $2.5 \%$ sodium hypochlorite solution $(20 \mathrm{~min})$, followed by three times washing in sterile distilled water (10 minutes each). Sterile seeds were germinated on B5 [16] hormone-free medium supplemented with $6.0 \mathrm{~g} / \mathrm{L}$ bactoagar (Spain). The $\mathrm{pH}$ of the media was adjusted to 5.5 prior the addition of agar and autoclaving at $121{ }^{\circ} \mathrm{C}$ for $20 \mathrm{~min}$. The seedlings were cultivated at a temperature of $25 \pm 2^{\circ} \mathrm{C}$ under a 16-hour photoperiod under 
illumination with cold white light with an intensity of 3000 lux (36 W, Philips) for three weeks.

\subsection{Induction of HR cultures}

Transformation of microshoots and three types of explants isolated from seedlings (hypocotyls, cotyledons and primary shoots of seedlings) was carried out using wild strains of $A$. rhizogenes A4-RT and 15834 SWISS. The strains were kindly provided by I.N. Kuzovkina (Institute of Plant Physiology of RAS, Moskow). All the explants were pricked with an insulin sterile syringe needle and placed in a B5 liquid nutrient medium containing a suspension of $A$. rhizogenes (48-hour grown in YEB liquid medium up to optical density at $\mathrm{OD}_{600}=0.4$ ). Control explants were also pricked with an insulin syringe needle and placed in B5 liquid bacteria-free culture medium. After 24 hours or 48 hours of incubation in a bacterial suspension, the explants were washed with sterile medium, blotted dry on filter paper and transferred on agar-solidified $\mathrm{B}_{5}$ hormone-free medium containing $500 \mathrm{mg} /$ L Ceftriaxone (Rafarma, Russia) to eliminate the residual bacteria. After 30 days of cultivation, the transformation frequency was evaluated as a ratio of the number of transformed explants to their total number. The selection of HR formed on the explants was carried out visually according to phenotypic characteristic (lateral branching, lack of geotropism, ability to grow on a hormone-free environment) [17]. The following two passages, the roots were cultured on hormone-free agarized $\mathrm{B}_{5}$ medium containing a reduced dose of the antibiotic $(250 \mathrm{mg} / \mathrm{L})$ to completely eliminate the bacteria. Each root explant was propagated as a separate line. The period between the passages was 4 weeks.

\subsection{PCR confirmation of transformation}

To confirm the transgenic status of HR culture, PCR analysis was performed for rol $\mathrm{C}$ and rol B genes. To reveal possible contamination with agrobacterial DNA, the preparations were tested by amplification the sequence of vir $\mathrm{C}$ gene. The total genomic DNA was isolated according to the protocol of the commercial DiamondDNA Plant Kit D kit (DiamondDNA, Russia). Non-transformed A. membranaceus roots DNA were used as a negative control. Ri-plasmid (pRi) DNA A. rhizogenes 15834 SWISS isolated with Plasmid Miniprep set (Evrogen, Russia) was applied as a positive control. The following pairs were used as primers for detecting: for rolB (5'-CAATGGATCCCAAATTGCTATTCC-3' and 5'-CGGCTTTAGGCTTCTTTCTTGAGG-3'); for ATGGCTGAAGACGACCTGTGT-3' and 5'-TAGCCGATT GCAAACTTGCAC-3'); for $\operatorname{virC}$ (5'-ATCATTTGTAGC GACT-3' and 5'-AGCTCAAACCTGCTTC-3'); PCR was carried out in a thermal cycler C-1000 (Bio- Rad, USA). The final volume of the reaction mixture was $25 \mu \mathrm{L}$ : $10 \mathrm{ng}$ of DNA, $0.4 \mu \mathrm{M}$ of each primer (Evrogen, Russia), $0.25 \mathrm{mM}$ of each dNTP (Evrogen, Russia), 1 unit, HS Taq DNA polymerase (Evrogen, Russia), $2.5 \mathrm{mM}$ $\mathrm{MgCl}_{2}$ (Evrogen, Russia), and 1× Taq buffer (Evrogen, Russia). Amplification was carried out according to the following program: initial denaturation at $94{ }^{\circ} \mathrm{C}-3 \mathrm{~min} ; 35$ cycles (denaturation $94{ }^{\circ} \mathrm{C}-20 \mathrm{~s}$, primers annealing at $60^{\circ} \mathrm{C}$ for $\mathrm{rolB}$, virC and at $55^{\circ} \mathrm{C}$ for $\mathrm{rolC}$, elongation at $72{ }^{\circ} \mathrm{C}-60 \mathrm{~s}$ ); final elongation $-7 \mathrm{~min}$ and storage at $4{ }^{\circ} \mathrm{C}$ until further use. The amplification products were stained with $3 \times$ SYBR- Green (Medigen, Russia) and separated in $1.7 \%$ agarose gel in $1 \times$ TAE-buffer at voltage $2.7 \mathrm{~V} / \mathrm{cm} 2$. Gels were visualized and recorded by the GelDoc-It imaging system (Bio-Rad Laboratories Pty Ltd.). The amplified fragment sizes were determined using 100 bp DNA marker (Eurogen, Russia).

\section{Results}


Results on the frequency of transformation of explants isolated from A. membranaceus seedlings and in vitro derived microshoots showed a variation in this parameter depending on the type of explant, $A$. rhizogenes strain used and the duration of co-cultivation (Fig. 1).

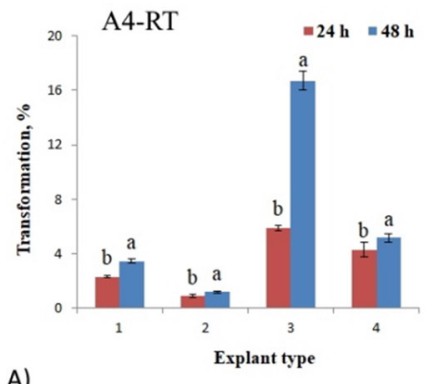

A)

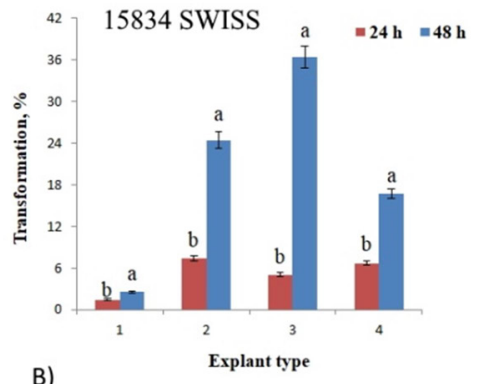

B)

Fig. 1. The transformation frequency (\%) in dependence on various types of A. membranaceus explants and time of co-cultivation with agrobacterial suspensions of A4-RT (A) and 15834 SWISS (B) strains. 1- hypocotyl, 2 - cotyledon, 3 - primary shoot, 4 - in vitro derived microshoot. Data is given as $\mathrm{M} \pm \mathrm{m}$. Means followed by the same letters do not differ significantly from each other according to Duncan's Means Separation test at $\mathrm{p} \leq 0.05$

It was established that the formation of HR of A. membranaceus depended on the type of explant. Low response to the action of bacteria of both strains was shown for hypocotyl seedlings, regardless of the time of treatment. For cotyledons, strain 15834 SWISS showed greater virulence, increasing the frequency of transformation by more than three times when the co-cultivation time lasted up to 48 hours. The transformation of the primary shoots of seedlings varied from 5.0 to $5.9 \%$ depending on the bacterial strain at $24 \mathrm{~h}$ of cocultivation. With an increase in the cultivation period to 48 hours, the frequency of transformation of primary shoots increased in several times. Thus, the induction of this type of explants using a suspension of strain 15834 SWISS for 48 hours increased the transformation rate by more than 7 times (up to $36.4 \%$ ) compared to 24 hours of treatment. The same strain was found to be the most effective for the transformation of microshoots, initiating the formation of HR with a frequency of up to $16.7 \%$ (Fig. 2, B). The primary shoots of seedlings of another species of astragalus, $A$. penduliflorus Lam, also showed a high level of transformation with strain 15834 SWISS [18]. Co-cultivation for 48 hours significantly increases the transformation efficiency of explants of many species, including Glycyrrhiza glabra, Linum mucronatum, and Artemisia annua [19, 20, 21]. Our data confirm the dependence of the frequency of HR formation on the virulence of the strain used [21]. Control explants (without infection with A. rhizogenes) cultured under identical conditions with transformed samples did not form roots.

The transgenic nature of obtained HR was confirmed by the PCR method with primers to agrobacterial oncogene $\operatorname{rolB}$, whereas the absence of agrobacterium contamination was examined with primers for $\operatorname{vir} C$ gene. Product formation of the expected length (Fig. 3) indicated a successful result of agrotransformation. PCR analysis using primers for the virC gene provided no bands corresponding to the size of virC from the HR lines, thus the absence of hybridization with vir probes indicated that samples were not contaminated with agrobacterium. 


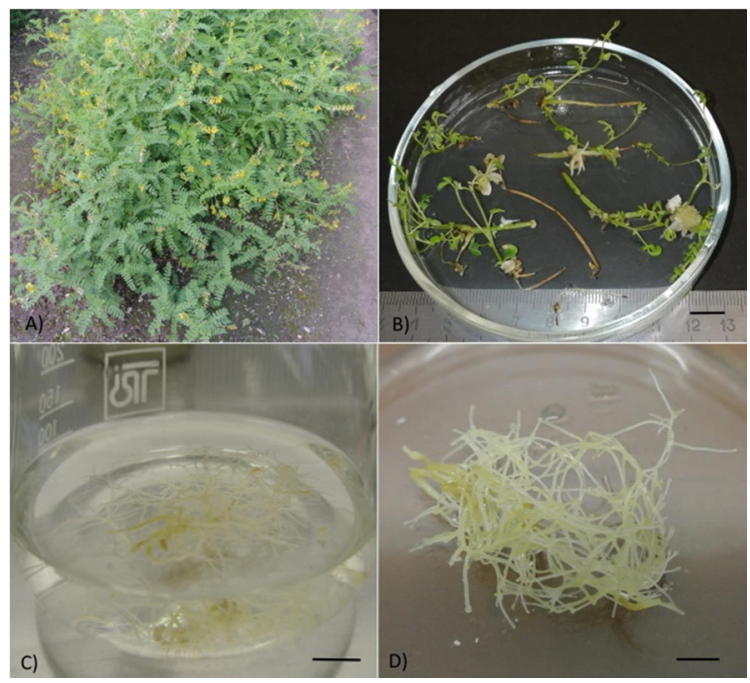

Fig 2. A. membranaceus in field collection (A); emergence of roots in microshoots after 30 days of inoculation with strain $A$. rhizogenes 15834 SWISS (B); HR of $A$. membranaceus cultivated for 4 weeks in liquid hormone free $\mathrm{B} 5$ medium (C); HR of $A$. membranaceus after 8 weeks of cultivation (D)

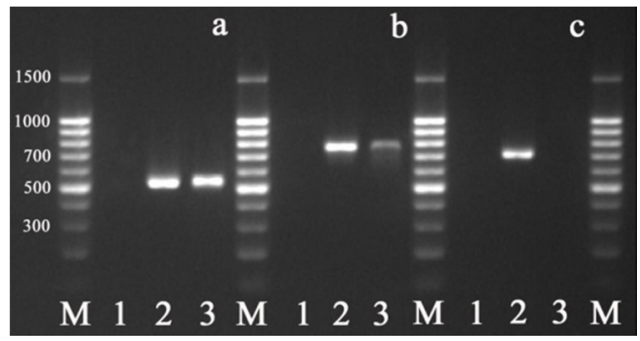

Fig.3. PCR detection of $\operatorname{rolC}(\mathbf{a}), \operatorname{rolB}(\mathbf{b}), \operatorname{virC}(\mathbf{c})$ genes. 1: total DNA of $A$. membranaceus nontransformed roots-negative control;2. Plasmid DNA of A. rhizogenes 15834 SWISS-positive control; 3. total DNA of the hairy root culture of A. membranaceus obtained by transformation of primary leaves with strain $A$. rhizogenes 15834 SWISS; $M$ - molecular weight marker $100 \mathrm{bp}$

In conclusion, our study provides the optimization of HR culture induction of $A$. membranaceus. It was revealed that primary shoots were the most responsive explant type. The best time for co-cultivation with a suspension of bacteria A. rhizogenes strain 15834 SWISS was 48 hours. PCR methods confirmed the successful transformation with primers to the agrobacterial oncogen in $\mathrm{rolB}$, as well as the absence of contamination with agrobacteria using primers for the virC gene. Subsequently, the obtained HR lines will be characterized by growth parameters and the content of the main pharmacologically active components.

The work was carried out within the framework of the state task of the Central Siberian Botanical Garden SB RAS No AAAA-A17-117012610051-5. In vitro material from the collection of the Central Siberian Botanical Garden SB RAS - USU 440534 "Collection of living plants indoors and outdoors" was used.

\section{References}

1. R. Verpoorte, A. Contin, J. Memelink, Phytochem. Rev. 1, 13 (2002) 
2. M.S. Hussain, S. Fareed, S. Ansari, M. Rahman, I.Z. Ahmad, M. Saeed, J. Pharm. Bioall. Sci. 4, 10 (2012)

3. E. McCoy, Progress in Drug Research. 65, 330 (2008)

4. A.M. Nosov, Biotechnology. 5, 8 (2010)

5. S. Chandra, R. Chandra, Phytochem. Rev. 10, 371 (2011)

6. A.B. Makhzoum, P. Sharma, M.A. Bernards, J. Trémouillaux-Guiller, Rec. Adv. Phytochem. 42, 95 (2013)

7. T. Isah, S. Umar, A. Mujib, M.P. Sharma, P.E. Rajasekharan, N. Zafar, A. Frukh, Plant Cell Tiss. Org. Cult. 132, 239 (2018)

8. T.A. Aseeva, K.F Blinova, G.P. Yakovlev, Lekarstvennye rasteniya tibetskoj mediciny (Novosibirsk, Nauka, 1985)

9. E. Bedir, N. Pugh, I. Calis, D.S Pasco, I.A. Khan, Biol. Pharm. Bull. 23, 834 (2000)

10. H.Y. Zhu, Y.Y. Zhang, G.Ye, Z.X. Li, P. Zhou, C.G. Huang, Biol. Pharm. Bull. 32, 68 (2009)

11. J.Y.W. Chan, F.C. Lam, P.C. Leung, C.T. Che, K.P. Fung, Phytother. Res. 23, 658 (2009)

12. I. Ionkova, A. Shkondrov, I. Krasteva, T. Ionkov, Phytochem. Rev. 13, 343 (2014)

13. A. Napolitano, S. Akay, A. Mari, E. Bedir, C. Pizza, S. Piacente, J. Pharm. Biomed. Anal. 85 (2013)

14. J. Fu, Z. Wang, L. Huang, S. Zheng, D. Wang, S. Chen, H. Zang, S. Yang, Phytother. Res. 28, 1275 (2014)

15. T. Murashige, F. Skoog, Physiol Plant, 15, 473 (1962)

16. O.L. Gamborg, D.E. Eveleigh, Can. Journal of Biochemistry. 46, 417 (1968)

17. D. Tepfer, Cell. 37, 959 (1984)

18. E.V. Ambros, O.V. Kotsupy, T.A. Kukushkina, T.V. Zheleznichenko, T.I. Novikova, Chemistry of plant raw material. 2 (2020)

19. A. Samadi, J. Carapetian, R. Heidari, M. Jafari, A. Hassanzadehgorttapeh, Not. Bot. Horti. Agrobo. 40, 125 (2012)

20. A. Giri, C.C. Giri, V. Dhingra, M.L. Narasu, Nat. Prod. Lett. 15, 229 (2001) 